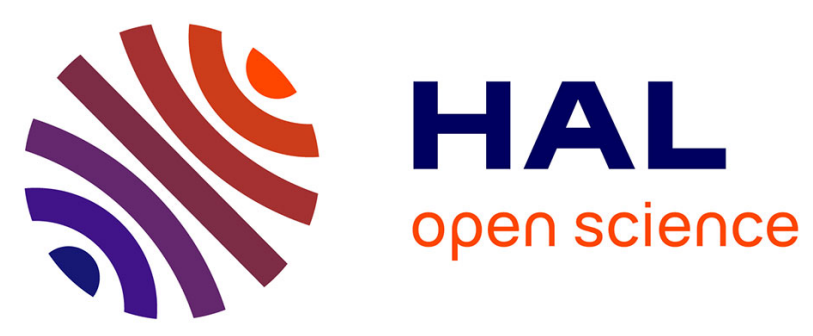

\title{
Liquid Crystal-Mediated 3D Printing Process to Fabricate Nano-Ordered Layered Structures
}

\author{
Ali Rouhollah Jalili, Alexandra Satalov, Sahar Nazari, Bryan Harry Rahmat \\ Suryanto, Jing Sun, Mohammad Bagher Ghasemian, Mohannad Mayyas, \\ Ahmad Kandjani, Ylias Sabri, Edwin Mayes, et al.
}

\section{To cite this version:}

Ali Rouhollah Jalili, Alexandra Satalov, Sahar Nazari, Bryan Harry Rahmat Suryanto, Jing Sun, et al.. Liquid Crystal-Mediated 3D Printing Process to Fabricate Nano-Ordered Layered Structures. ACS Applied Materials \& Interfaces, 2021, 13 (24), pp.28627-28638. 10.1021/acsami.1c05025 . hal03367907

\section{HAL Id: hal-03367907 https://hal.science/hal-03367907}

Submitted on 28 Oct 2021

HAL is a multi-disciplinary open access archive for the deposit and dissemination of scientific research documents, whether they are published or not. The documents may come from teaching and research institutions in France or abroad, or from public or private research centers.
L'archive ouverte pluridisciplinaire HAL, est destinée au dépôt et à la diffusion de documents scientifiques de niveau recherche, publiés ou non, émanant des établissements d'enseignement et de recherche français ou étrangers, des laboratoires publics ou privés. 


\title{
Liquid crystal mediated 3D printing process to fabricate nano-ordered layered structures
}

Ali (Rouhollah) Jalili ${ }^{*}$, Alexandra Satalov ${ }^{2}$, Sahar Nazari ${ }^{1}$, Bryan Harry Rahmat Suryanto ${ }^{3}$, Jing Sun ${ }^{1}$, Mohammad Bagher Ghasemian ${ }^{1}$, Mohannad Mayyas ${ }^{1}$, Ahmad E Kandjani ${ }^{4}$, Ylias M Sabri ${ }^{4}$,Edwin Mayes ${ }^{4}$, Suresh K. Bhargava ${ }^{4}$, Jun Araki ${ }^{5,6}$, Cécile Zakri ${ }^{7}$, Philippe Poulin ${ }^{7}$, Dorna Esrafilzadeh ${ }^{*}$, Rose Amal $^{1}$

1- School of Chemical Engineering, University of New South Wales (UNSW), Sydney, NSW 2052, Australia

2- Institut für Anorganische Chemie, Leibniz Universität Hannover, Callinstr. 9, 30167 Hannover, Germany

3- Australian Centre for Electromaterials Science, School of Chemistry Monash University, Clayton Victoria 3800, Australia.

4- School of Science, RMIT University, Melbourne, VIC, 3001, Australia

5- Faculty of Textile Science and Technology, Shinshu University, Tokida 3-15-1, Ueda, Nagano prefecture, 386-8567, Japan

6- Institute for Fiber Engineering (IFES), Interdisciplinary Cluster for Cutting Edge Research (ICCER), Shinshu University, Tokida 3-15-1, Ueda, Nagano prefecture, 386-8567, Japan

7- Centre de Recherche Paul Pascal - CNRS, University of Bordeaux, 33600 Pessac, France

8- Graduate School of Biomedical Engineering, University of New South Wales, Sydney, NSW, 2031 Australia

*Corresponding author: Email: ali.jalili@unsw.edu.au; d.esrafilzadeh@unsw.edu.au

\begin{abstract}
The emergence of three-dimensional (3D) printing promises a diasruption in the design and on-demand fabrication of smart structures in applications ranging from functional devices to human organs. However, the scale at which 3D printing excels is within macro and microlevels, principally lacks the spatial ordering of building blocks at nano-levels, which is vital for most multi-functional devices. Herein we employ liquid crystal (LC) inks to bridge the gap between the nano and micro-scales in a single-step 3D printing. The LC-ink is prepared from
\end{abstract}


mixtures of liquid crystals of nanocellulose whiskers and large sheets of graphene oxide, which offers a highly ordered laminar organization not inherently present in the source materials. Liquid crystal mediated 3D printing imparts the fine-tuning required for the design-freedom of architecturally layered systems at the nanoscale with intricate patterns within the $3 \mathrm{D}$ printed constructs. This approach empowered the development of a high-performance humidity sensor comprising of self-assembled lamellar organization of NC whiskers. We observed that the NC whiskers that are flat and parallel to each other in the laminar organization allow facile mass transport through the structure, demonstrating a significant improvement in the sensor performance. This work exemplifies how LC-ink, implemented in a 3D printing process, can unlock the potential of individual constituents to allow macroscopic printing architectures with nanoscopic arrangements.

\section{Keywords}

3D printing, liquid crystalls, nanocellulose, self-orientation, humidity sensor

\section{Introduction}

Additive manufacturing (AM), also known as 3D printing, is becoming increasingly dominant in advanced materials processing and application. It provides the ability to design and prototype geometrically complex structures that integrate new functionalities ${ }^{1-4}$. This technology enables the production of structured functional materials and can close the gap between new materials discoveries and manufacturing ${ }^{5}$. However, to increase the freedom around materials designs, we should control the arrangement of building blocks form macro-scale to molecular levels. If this is achieved, 3D printing techniques can converge into the nanoscale realm, while the properties of individual building blocks can be used with ultimate efficiency. Nevertheless, conventional 3D printing inks and approaches such as those using thermoplastic polymers, UV curable resins, and metals are not designed to control the distribution of materials at submicron levels. To address this challenge, researchers have developed composites inks based on nanoscale materials to $3 \mathrm{D}$ print structures with improve performance and obtain new functionalities ${ }^{6-9}$. Similar strategies may allow 3D printing to go beyond the typical zresolution (which is conventionally $>10$ s of $\mu \mathrm{m}$ ) and control the nanoscale $z$-slicing planarity (called Znano here). The same concept can also be similarly applied to Xnano-ynano planes. Such a multi-scale manufacturing technique can print in microscale in $3 \mathrm{D}\left(\mathrm{x}_{\mu}\right.$, 
$\left.\mathrm{y}_{\mu}, \mathrm{z}_{\mu}\right)$ whilst fine-tuning the planar distribution of the building blocks in nanoscale $3 \mathrm{D}$ domains (Xnano, Ynano, Znano) (Figure. 1).

Biological systems implement anisotropic molecular building blocks and their remarkable selfassembling properties to achieve liquid crystal (LC) mediated complex structural arrangements and patterns ${ }^{10-12}$. Anisotropic nanomaterials (e.g. prisms, rods, cubes, and platelets) can also support self-ordering which resembles liquid crystalline behaviour ${ }^{13}$. These systems are desirable due to the possibility of solution processing of liquid crystals to form ordered structures of those nanomaterials ${ }^{14}$. To this end, there have been many reports harvesting the dynamic conformations controlled by anisotropic building blocks to put together nanoscale components to functional microscopic ordered systems adequately. For example, LC phases from graphene oxide (GO), carbon nanotube, nanocellulose (NC) and $\mathrm{MoS}_{2}$ have been widely used for extrusion-based fabrication processes such as fibre spinning ${ }^{15-20}$. In $3 \mathrm{D}$ printing, direct ink writing has been the most common extrusionbased additive manufacturing technique used for 3D printing of such colloidal inks and liquid crystals ${ }^{21-23}$. However, in most cases, to facilitate the printing process, polymers and binders have been added to the ink to modify the flow behaviour and provide printability ${ }^{23-25}$.

Nevertheless, most liquid crystals are sensitive to changes in the ionic strength of the dispersions that can occur due to the additives ${ }^{26}$. Therefore, there is a need to stabilize the LC phase and facilitate the integration of the liquid crystal mediated systems with additive manufacturing. This can result in achieving a new printable LC ink that can carry forward the inherent LC order and, at the same time, maintain the properties of the constituent materials.

Inspired by the classical theory of the biaxial nematic phases of micellar systems ${ }^{27}$, we believe a stabilized LC phase can be formulated via binary mixtures of two uniaxial phases of rod-like (1D) and plate-like (2D) liquid crystalline phases. Here, in the case of rod-like material, we select nanocellulose whiskers (NC whiskers). Cellulose and its industrial wastes can be regenerated as an available natural product known as nanocellulose $(\mathrm{NC})^{28}$. Although envisaged as a ground-breaking technological material for use in biomedical, optical, energy, green composites, and environmental applications ${ }^{29-31}$, NC has yet to live up to its full potential. Due to their anisotropic nature and chirality 32, 33, NC whiskers can form a chiral nematic liquid crystalline phase at sufficiently high 
concentration. In the case of the second component, a liquid crystalline phase comprising super-flexible two-dimensional (2D) ultra-large graphene oxide (GO) sheets (average lateral dimensions of $37 \mu \mathrm{m} \pm 23 \mu \mathrm{m}$ and thickness of $0.8 \mathrm{~nm})^{34}$ is used; to induce an additional order parameter coming from these platelets-like particles. Such an all-around process of mixing stiff( $\mathrm{NC}$ whiskers) and flexible elements with LC medium can impart the fine-tuning needed for the design-freedom of architecturally complex systems at the sub-micron level. We show that this ink allows intricate patterns and functionality in each layer. We show the capability of 3D printing of nano-ordered structures in an example by developing an intelligent material for humidity sensing from NC whiskers with unequivocal performance outperforming the conventional and state-of-the-art sensors.

\section{Results}

\section{LC-ink formulation}

The geometrical structure of our NC whiskers was analyzed by small-angle X-ray scattering (SAXS) experiments. Modelling of low concentration suspensions of NC carried out using elliptical cylinders shaped with the following parameters: lengths, axis ratios, and minor radia of 47, 7, and $2.1 \mathrm{~nm}$, respectively (Figure $\mathrm{S} 1$ ). These parameters are in agreement with the geometrical features of particular $\mathrm{NC}$ whiskers produced from the hydrolysis of cotton reported in the literature ${ }^{35}$. 
A

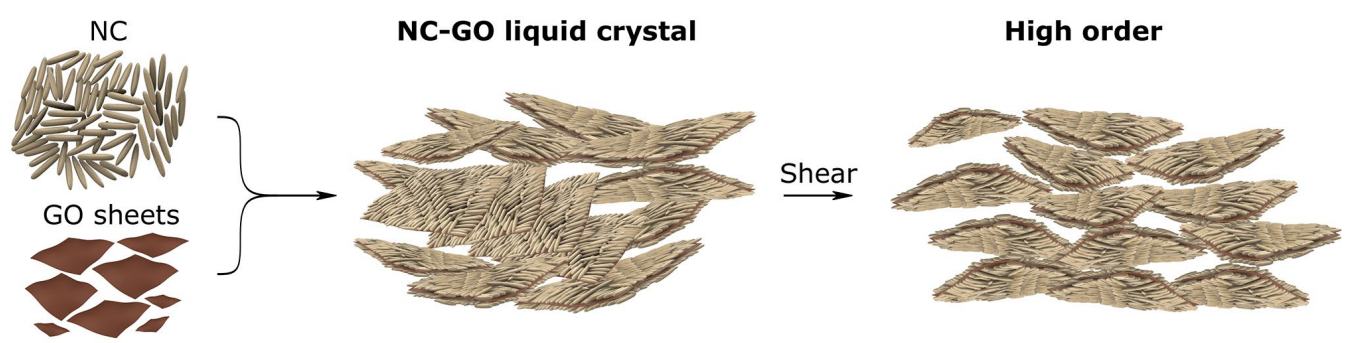

B

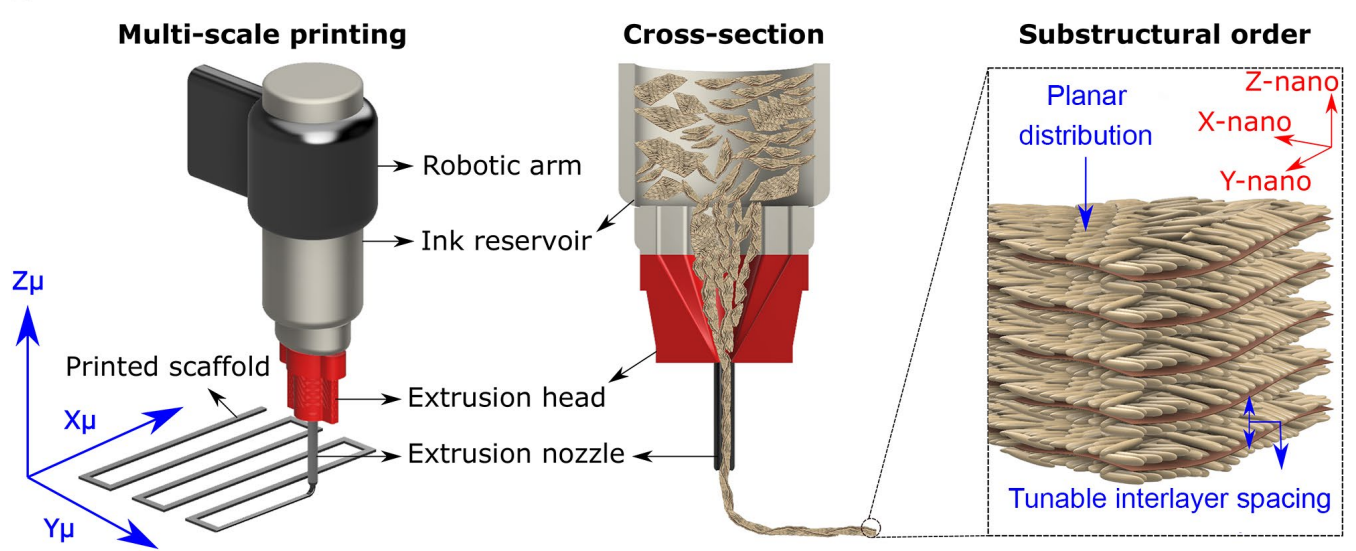

Figure. 1. The step-by-step illustration of our strategy on the liquid crystal mediated 3D printing process. (A) Schematic diagram of ink preparation with LC characteristics. The addition of ultra-large GO sheets to LC of NC whiskers modifies the local environment to exhibit a percolating active network resembling the biaxial LC phase of rod and plate mixtures that are ordered long the shear field. (B) Schematic illustration of 3D printing of nano-ordered layered structures (multi-scale 3D printing concept). In a combined top-down and bottom-up approach, 3D printing and LC-ink utilized to print at 3D micro-level and 3D nano-level orderings. The 3-dimensional coordinates at the microscale $\left(x_{\mu}, y_{\mu}, z_{\mu}\right)$ control via the printer robotic arm, while the layer-by-layer ordered of the LC-ink fine-tunes the spatial order at the 3dimensional nanoscale $\left(\mathrm{x}_{\mathrm{nano}}, \mathrm{y}_{\text {nano, }}, \mathrm{z}_{\mathrm{nano}}\right)$. Each GO sheet represents an $\mathrm{x}_{\text {nano }}-\mathrm{y}_{\text {nano }}$ plane. Therefore, the controlled glass formation and homogenous intercalation of the NC particles between the GO layers, while maintaining the original nematic order is key to engineer the $\mathrm{x}_{\text {nano- }}$ $y_{\text {nano }}$ plane. Here, we trigger $z_{n a n o}$-slicing in ink (by adding GO) while controlling the gel-like glass formation by essentially freezing the nematic ordering of NC between the GO sheets in each $\mathrm{x}_{\text {nano, }} \mathrm{y}_{\text {nano }}$ plane.

Due to the anisotropic nature and chirality of the NC whiskers $32,33,36$, they support the formation of chiral nematic LC phase (helical) in the aqueous media (also see supporting section 1). Polarised light optical microscopy (POM) analysis of NC dispersions in 
capillary tubes, filled with suspensions of increasing volume fractions after being stationary for a month, reveals the following features. At volume fractions $\phi<0.6 \mathrm{vol} \%$, the suspensions are isotropic liquids. At $0.6 \mathrm{vol} \%<\phi<1.5 \mathrm{vol} \%$, the suspensions are biphasic with a phase separation between the denser birefringent phase at the bottom and the isotropic one at the top. As expected, the proportion of the birefringent phase gradually increases with the overall nanocellulose volume fraction until $\phi>1.5 \%$ vol, where no separation is observed.

At $\phi>\sim 3$ vol\%, the NC suspension forms a gel-like phase, which was used to test the 3D printability process. However, because the NC whiskers show a tendency to form bundles during casting and the subsequent drying (Figure. 2 A-B), direct ink writing of particularly fine structures was not possible (Figure $2 \mathrm{C}, \phi=3$ vol\%). However, a further increase in the concentration (higher viscosity) could not provide a significant printability (Figure. 2 D-E). Even at $\phi=\sim 8$ vol\% which was the highest concentration that we could obtain (via centrifugation), the printed scaffolds were prone to mechanical failures and could not support fine structures with a thickness higher than $\sim$ few millimetres. Moreover, the cross-section of the freeze-dried (Figure 2 F) and air-dried (Figure $2 \mathrm{G}$ ) printed structure show no evidence of a long-range orientation. It appears that the inherent order of the LC phase could not be preserved via the printing process and the subsequent drying. Please note, the difference between the thickness of a typical sample that prepared via freeze-drying is at least 10 times greater than an air-dried sample. To further analyze the long-range order of the $\mathrm{NC}$ whiskers in each printed filament, POM and AFM analysis were employed (Figure $2 \mathrm{G}-\mathrm{H}$, respectively), which further confirm the lack of long-range order in the printed samples.

Subsequently, the printing ink was modified by the addition of a small amount of ultralarge amphiphilic GO to create the so-called shape amphiphiles (Figure. 3A-B). Here, the NC whiskers are stabilized through interactions between the oxygen functional groups on top of the GO sheets and sulphate ester groups on the NC whiskers. Even trace amounts of ultra-large $\mathrm{GO}$ sheets $\left(\phi_{\mathrm{GO}}=0.018 \mathrm{Vol} \%\right)$ modify the thermodynamic and rheological properties of the fluid, potentially forming highly ordered biaxial LC phases that are predicted theoretically for mixtures of rods and plates (see supporting section 3-4) 27,37 . 
In theory, mixtures of nano-rod-like and nano-plate-like particles support the formation of a stable new phase so called biaxial LC phase ${ }^{38-40}$. In a biaxial phase, both the rodlike and the plate-like particles are orientationally ordered, the axis of alignment of the rod being perpendicular to that of the plates. If such a phases formed, it should result in achieving an efficient separation of individual particles to avoid aggregation and phase separation without paying the penalty of losing the LC order in ink, and consequently, no failures, defects, bundling and randomization during the printing process. However, the challenge still exists as there is no experimental proof of the miscibility and long term stability of the mixtures of rods and disks at high rod concentrations as these systems tend to phase separate ${ }^{39-41}$. The shape incompatibility of rods and disks, which is the direct result of large translational entropy in the phase-separated state, plays a detrimental role leading to the failure of previous attempts to induce self-organization and long-range ordering in most mixed LC systems ${ }^{39-41}$. 

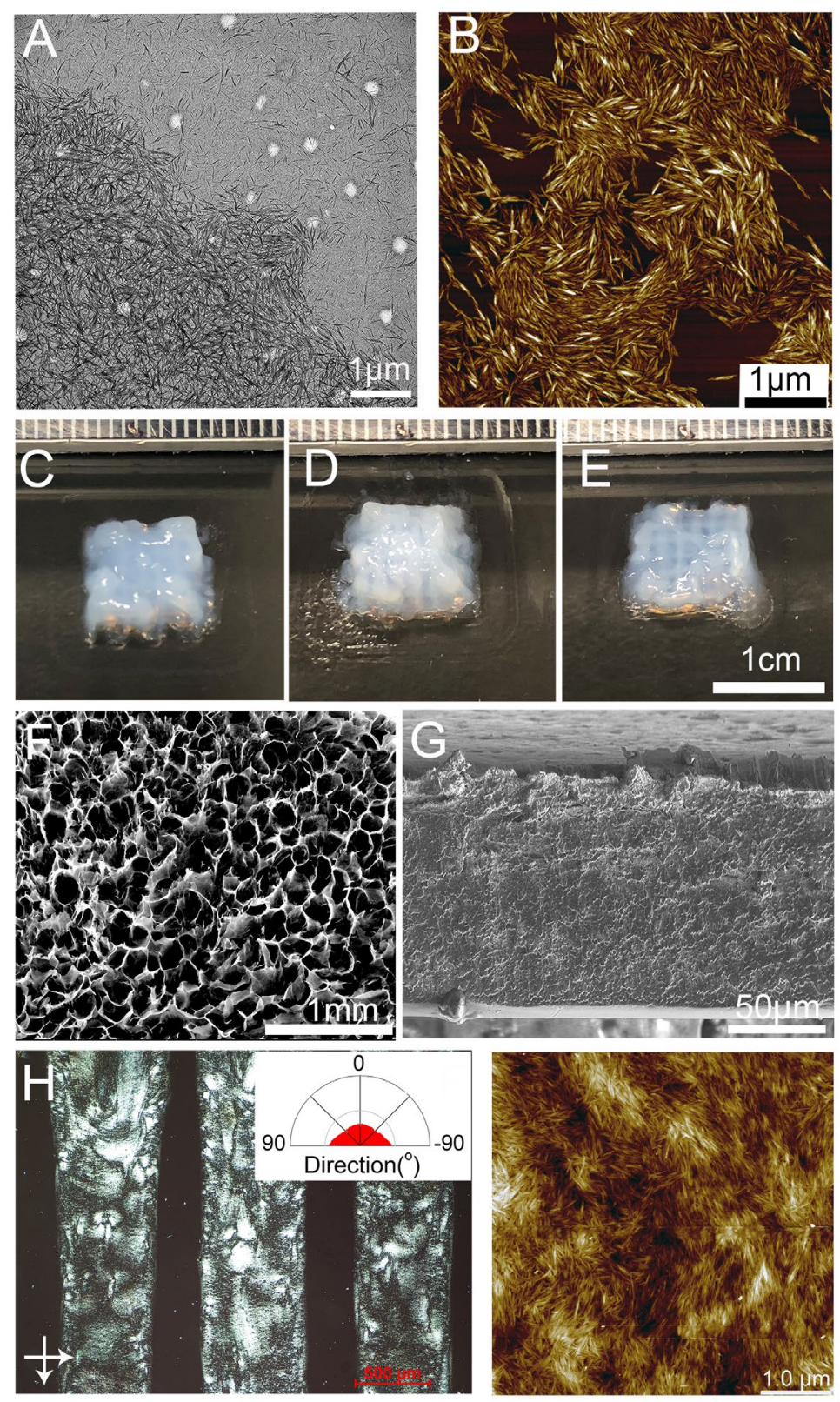

Figure. 2. Bundle formation and randomization of NC whiskers. (A-B) NC whiskers form aggregates and randomly oriented bundles as depicted in TEM and AFM micrographs, respectively. (C -E) Photo of as-printed scaffold made from pure NC whiskers at various concentrations of $\phi=3 \mathrm{Vol} \%$, $\phi=5 \mathrm{Vol} \%$ and $\phi=8 \mathrm{Vol} \%$, respectively. (F-G) SEM micrograph of the cross-section of a freeze-dried and air-dried scaffold, respectively. (H) A POM image of printed filament from pure NC whiskers, $\phi=8$ Vol\%. Arrows show the orientation of polariser and analyzer. Inset shows a histogram of orientation (HoG descriptor), suggesting no preferential order of the LC domains in the printed tracks. The HOG descriptor counts occurrences of gradient orientation in the POM image. (I) AFM micrograph further confirming the random distribution of $\mathrm{NC}$ whiskers on the surface of the filaments. 

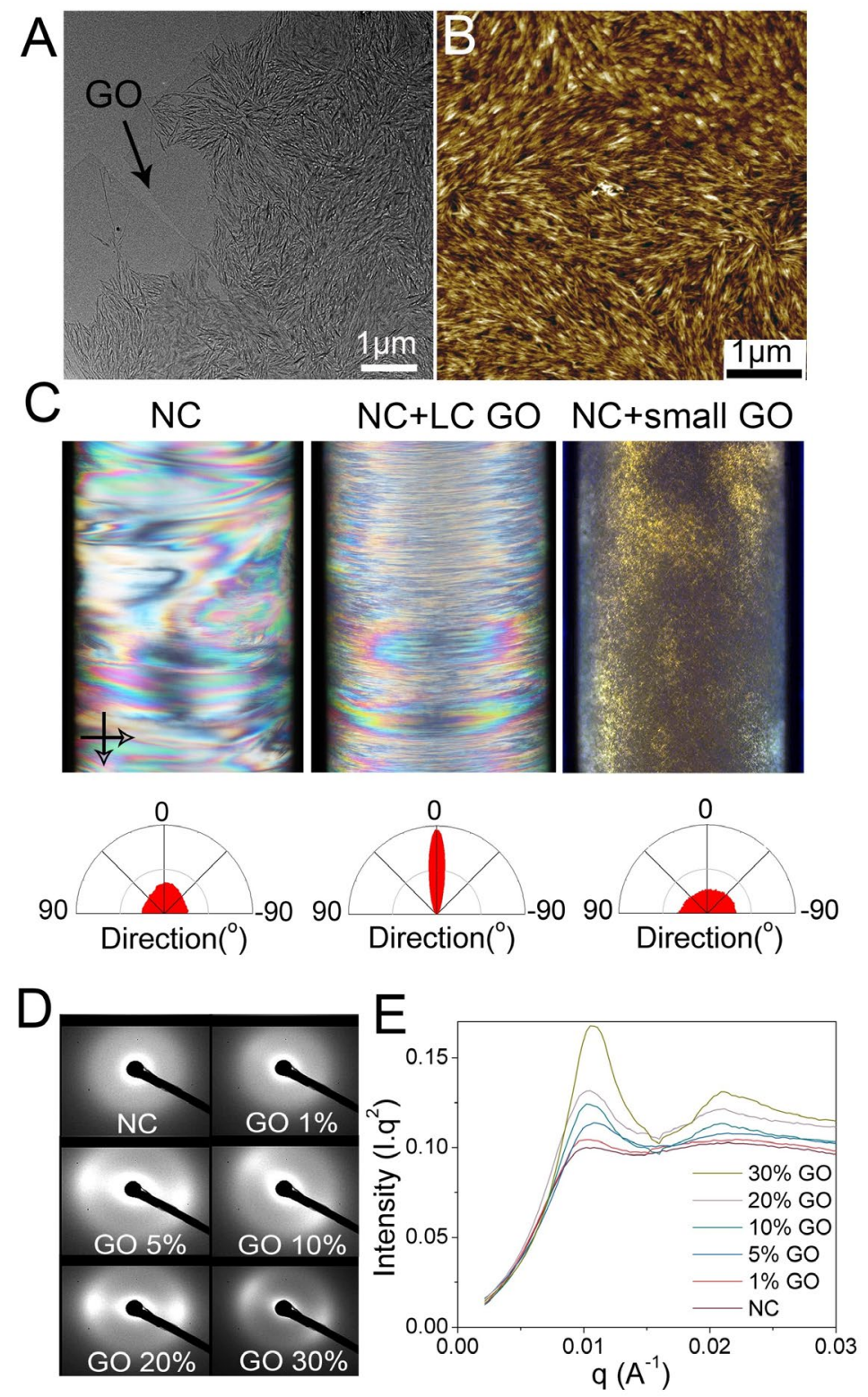

Figure. 3. The Structural Evolution of LC phase upon the addition of GO. (A) TEM and (B) AFM micrographs of GO-NC binary showing how ultra-large GO sheets (LC GO) can act as a template to minimize randomization and bundling of NC whiskers. (C) POM micrographs of samples in capillary tubes (arrows show the orientation of polariser and analyzer). Employing NC whiskers on their own and mixed with small GO (non-LC GO, the lateral sheet size $<1 \mu \mathrm{m}$, which is prepared by $1 \mathrm{~h}$ bath sonication of the large sheets) do not result in the orientation of the nematic phase. On the other hand, the addition of even trace amounts of ultra-large GO sheets $\left(\phi_{\mathrm{GO}}=0.018 \mathrm{Vol} \%\right.$, the lateral sheet size $>$ $10 \mu \mathrm{m}$,) generates enough excluded volume effect to transform the LC phase to a unidirectionallyordered nematic phase. HoG descriptor from the POM images is also presented for these samples. In contrast to the NC whiskers, the LC domains of the ultra-large GO sheets added samples show a global orientation. (D) Small-angle X-ray scattering (SAXS) diffractions and (E) Lorentz-corrected curves of 
dispersions as a function of GO ratio $\left(\phi_{\mathrm{NC}+\mathrm{GO}}=2 \mathrm{Vol} \%\right)$. The noted GO percentage in each image is the volume ratio of $\mathrm{GO}$ vs. $\mathrm{NC}$ whiskers.

Interestingly, such phenomena do not occur in our binary LC system (please see section 6 in the supporting information). The key here is regarding exploiting the selforientation in a binary system involving entities from nanometer to micrometre lengthscale with close density while having a large difference in their aspect ratios $(\sim 50,000$ for GO vs. $\sim 100$ for $\mathrm{NC}$ ).

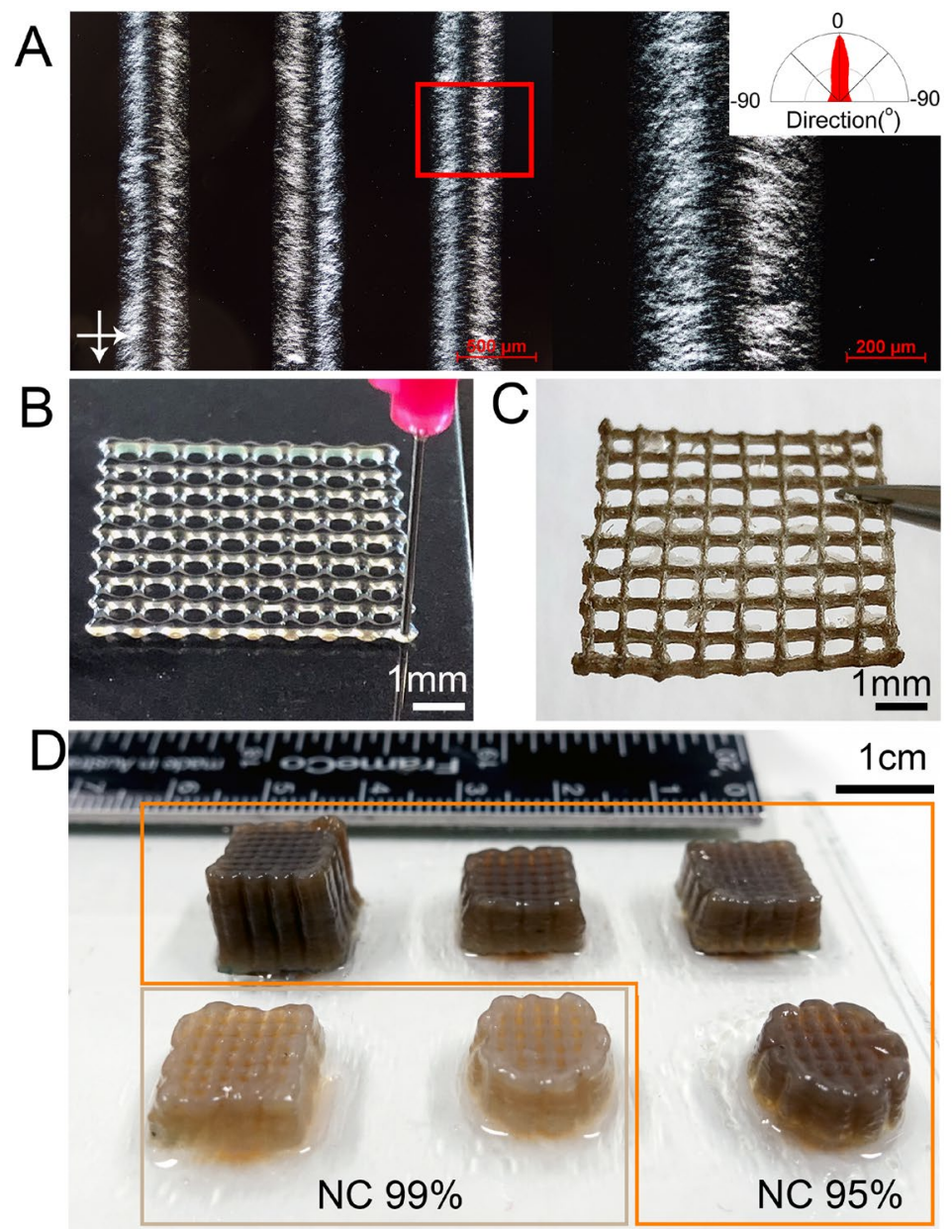

Figure. 4. Highly improved printing properties using the binary LC ink. (A) POM images of printed tracks from LC-ink (arrows show the orientation of polariser and analyzer). HoG descriptors (inset) from the POM images show much improved ordered LC domains in the printing path of the LC ink. (B) A picture of a printed grid after the deposition of the first layer. After deposition of 10 layers, the dried scaffold was mechanically robust, allowing removing from the substrate, as evidenced by micrograph in (C). (D) As-printed 3D scaffolds with different shapes, hight and GO content (1\% and $5 \%$ GO content). After deposition of 100 layers, 
the hight of $\sim 1 \mathrm{~cm}$ was achieved. Please note the addition of even $1 \% \mathrm{GO}\left(\phi_{\mathrm{GO}}=0.018 \mathrm{Vol} \%\right)$, resulted in much-improved printability.

The birefringence property of NC whiskers, non-LC GO and LC GO added NC whiskers in a capillary tube was investigated by employing POM images (Figure. $3 \mathrm{C}$ and also see supporting section 5-6 when samples observed from two different viewpoints). It appears that LC GO sheets restructure the alignment of the $\mathrm{NC}$ whiskers by reconfiguring their principle axes perpendicular to the capillary tube. As such, LC GO sheets with an aspect ratio much higher than the NC whiskers evolve the random distribution of nematic domains into an orderly laminar organization. The improvement in the orientation of $\mathrm{NC}$ whiskers is also verified using small-angle X-ray scattering (SAXS) study (Figure. 3 D-E), as the peak at lower q value becomes stronger, highlighting the long-range ordering induced by the addition of LC GO sheets. Moreover, as the volume fraction of LC GO increases (constant solid content of $\phi_{\mathrm{NC}+\mathrm{GO}}$ of $2 \mathrm{Vol} \%$ ), the peak does not move toward higher q values indicating that the repeating distance of particles in the dispersions stays the same, meaning no agglomeration/segregation in the system.

\section{Printing self-ordered structures}

POM analysis was similarly employed to study the sub-structural ordering that occurred throughout the printing process (Figure. 4 A, and also see supporting section 5). The asprinted tracks containing 1\% LC GO sheets (from here we refer as GO) demonstrate much-improved printability while demonstrating significant orientation in the birefringence property. Interestingly, such small amount of GO $\left(\phi_{\mathrm{GO}}=0.018 \mathrm{Vol} \%\right)$ could self-control the LC phase and thus the 3D printability of the NC dispersions (Figure. 4 B-D), avoiding excessive use of additive (see also supporting section 7). 

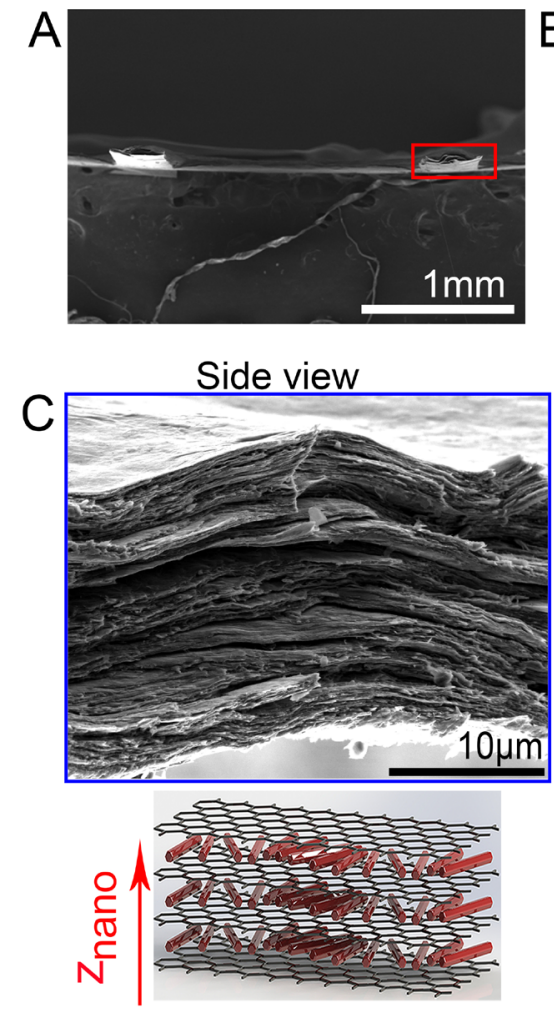

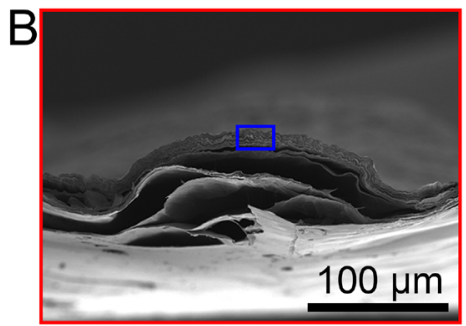

Top view

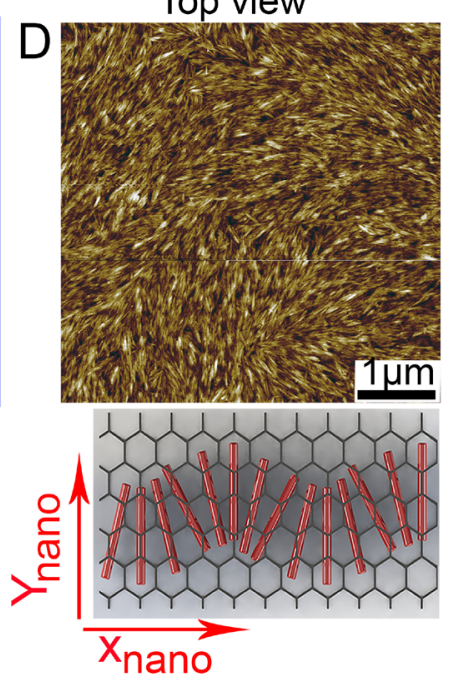

Figure. 5. Nano-ordered printed filaments using the binary LC ink. (A-C) SEM analysis of the cross-section of a printed filament containing 1\% LC GO at different magnifications. (D) AFM of the surface of the filament shows the orderly distribution of the NC whiskers on the surface (at $\mathrm{x}_{\text {nano }}$ and $\mathrm{y}_{\text {nano }}$ plane).

Figure 5 A-C shows cross-sectional SEM images of a typical printed grid (one layer) demonstrating the internal structure of each printed filament. The self-rearrangement of large GO sheets induces long-range ordering to the ink that was carried over through the $3 \mathrm{D}$ printing and subsequent drying processes. In other words, the evolution of the nematic phase to highly ordered LC-ink allows the control over the layer-by-layer organization (lamellar organization) and subsequent distribution of NC whiskers in the Xnano-ynano planes during the extrusion process (Figure. 5D).

Upon the phase modification using LC GO, a semi-permanent gel-like phase (network) formed ${ }^{42,43}$, that could support the extrusion printing process. It appears that the large difference in the aspect ratio of the particles forced the NC whiskers to be trapped in an electrostatic cage by the repulsive long-range forces exerted by large sheets of LC GO. 
Furthermore, as the system trapped in metastable kinetic traps and cannot relax toward a randomized state, the original nematic order maintained in the final printed sample. In the nematic phase, each component can interact with or get feedback from other neighbouring components to respond to local environmental stimuli such as shear flow. In the LC ink, the configurational entropy and excluded volume effects, due to the large anisotropic shape of the constituents, magnifying this effect. However, the non-LC GO ink could not generate enough excluded volume to underpin such a gel-like network (Figure S6, also see supporting information section2). This could explain why in 3D printing of non-LC graphene-based composites a circular orientation of the particles parallel to the nozzle wall occurs ${ }^{44}$. Similarly, inducing a layer-by-layer order to nonLC rod shape materials, such as single walled nanotube (SWNTs) dispersions, requires much larger quantities of LC GO (minimum 90 vol\%) to achieve a percolating network and subsequently the layer-by-layer order ${ }^{14}$.

To further corroborate the conjecture of the lamellar organization, we also performed top-view and side-view transmission and scanning electron microscope analysis (TEM and SEM). Cross-sectional SEM images of a freeze-dried printed paper (GO 1\%) evidences the layered organization at macro-scale (Figure. 6A-B). Cutting a thin slice by employing a focused ion beam (FIB) allowed analyzing the sample at much higher magnifications at two different angles (side-view and top-view, Figure. 6C-D, respectively). Cross-sectional TEM of the freeze-dried sample confirms the successful layered formation at nanoscale (Figure. 6C). Such a sandwich-like layered system restricts the randomization of the NC LC phase, dictating a reorganization of NC whiskers perpendicular to the main GO director in each layer during the printing process. Interestingly, in the top-view image (Figure. 6D) GO sheets are not visible. However, the presence of NC whiskers at different foci evidences the layer-by-layer organization. This is in contrast to the non-equilibrium glass formation in pure NC whiskers, which is hard to control ${ }^{33}$.

Our engineering capacity for glass formation, over altering the amount of GO present in the system, is also verified by studying the X-ray powder diffraction (XRD) patterns of the printed films (Fig. 6E). The lamellar organization is characterized by the dspacing between GO sheets using the XRD patterns. The d-spacing represents the interlayer spacing between individual GO sheets in the bulk material that is oriented 
perpendicularly to the diffraction plane. Printed films from pure NC whiskers represent two crystalline peaks at $2 \theta=15.3^{\circ}$ and $2 \theta=22.5^{\circ}$ (101 and 002 , respectively). When the LC-ink is employed, the XRD patterns of printed films signify the appearance of a $\mathrm{d}(001)$ peak at $2 \theta=9.1^{\circ}$ that gradually shifts towards higher $2 \theta$ as the GO content increases and reaches to $2 \theta=1.18^{\circ}$ for the LCGO sample (see also Fig. S16). The appearance of the $\mathrm{d}(001)$ peak of LCGO and systematic shift toward higher d-spacing verifies the controlled glass formation and homogenous intercalation of the NC particles between the GO layers. As such, we trigger a hierarchical layer development in the ink while controlling the gel-like glass formation by essentially freezing the desired level of layer-by-layer ordering and intrinsically limiting the distribution of $\mathrm{NC}$ whiskers in between the layers.

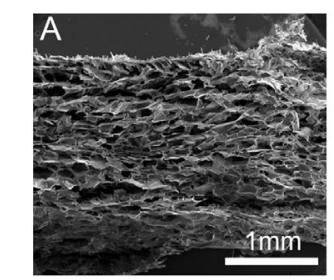

$\mathrm{E} \underset{3}{3}$

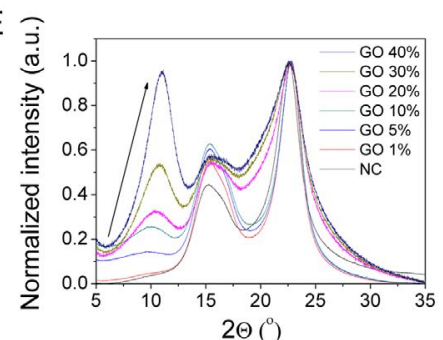

$\mathrm{H}$
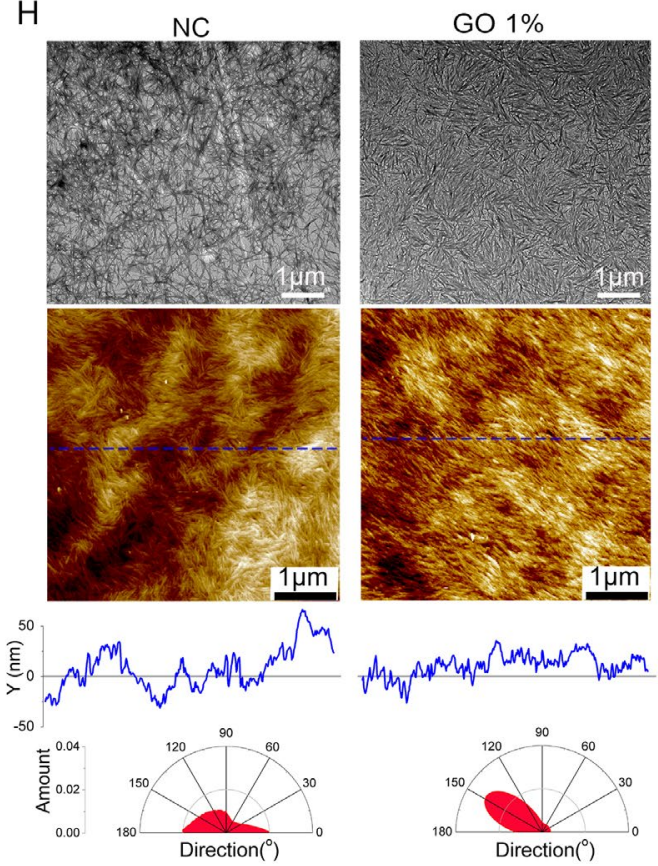

$\mathrm{F}$

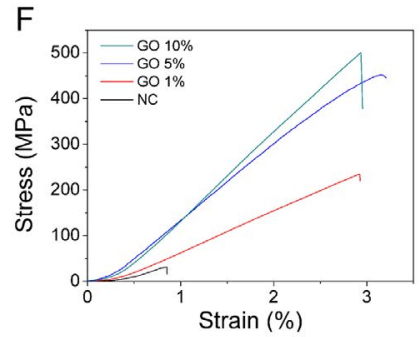

GO $5 \%$

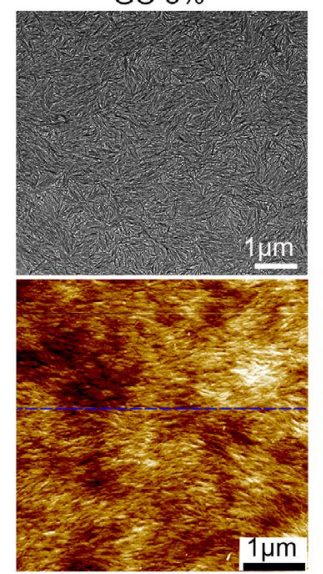

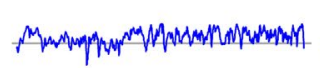
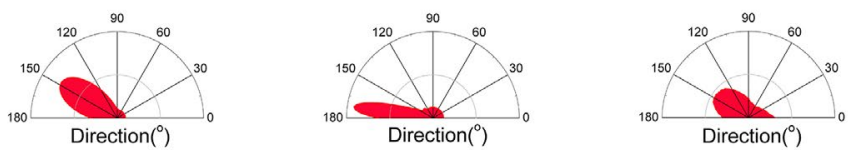
Figure. 6. Microscopic evaluation of the liquid crystal mediated printed architectures. (AB) Cross-sectional SEM images of a typical freeze-dried printed sample (LC GO 1\%) at two different magnifications, showing the ordering of LC GO-templated NC whiskers along with the nematic director (dipole of GO sheets). (C) Additionally, FIB cross-section of the freezedried sample (high magnification) shows the hierarchical layered structure $\left(Z_{\text {nano }}\right)$ due to the perpendicular orientation of NC whiskers on the GO sheets. (D). TEM image of the same sample at $90^{\circ}$ tilt (Top-view). It appears that the $\mathrm{NC}$ whiskers are embedded in between the GO sheets, as evidenced by the existence of out of focus and in-focus $\mathrm{NC}$ whiskers present in the micrograph. (E) X-ray powder diffraction (XRD) pattern of the printed structures. (F) Stressstrain curves of printed films as a function of GO content. (G) Halpin-Tsai model for composites containing ellipsoids was used to evaluate the elastic modulus of final architectures against the predictions for unidirectional and random distributions. Interestingly, the addition of even $1 \%$ GO results in perfect orientation leading to the highest possible elastic modulus for such systems. (H) Top-view TEM and AFM images highlighting the nanoscale ordering of printed films even after the subsequent solvent evaporation. Addition of even $1 \%$ ultra-large GO sheets transformed the arrangement of materials to a hierarchical layered structure while distributing the NC whiskers at $\mathrm{x}_{\text {nano }}$ and $\mathrm{y}_{\text {nano }}$ plane, as evidenced by the high profiles of the AFM images (blue lines). The maximum peak to valley of the height patterns increases from $83 \mathrm{~nm}$ for pure $\mathrm{NC}$ whiskers to $33.5 \mathrm{~nm}, 31 \mathrm{~nm}$, and $13.5 \mathrm{~nm}$ as $1 \mathrm{vol} \%, 5 \mathrm{vol} \%$, and $10 \mathrm{vol} \% \mathrm{GO}$ is added, respectively. Histogram of orientation (HoG descriptor) from the AFM images is also presented for each GO fraction.

Controlled distribution of $\mathrm{NC}$ whiskers perpendicular to the GO sheets drastically improved the mechanical properties without manipulating the molecular structure or applying excessive force, as evidenced by the stress-strain curves in Figure. 6F \& Table S1. We employ Halpin-Tsai equations for random and unidirectional orientation of ellipsoids to illustrate the orientation degree in the as-prepared dried samples. Interestingly, even the addition of $1 \% \mathrm{GO}\left(\phi_{\mathrm{GO}}=0.018 \mathrm{Vol} \%\right)$ to the system results in perfect orientation, leading to the attainment of maximum possible elastic modulus predicted for such a system (Figure. 6G). Our experimental data agreed well with the prediction based on unidirectional dispersion of GO sheets, verifying well-oriented layer-by-layer structure with a uniform distribution of materials across each plane, particularly for GO content lower than $10 \%$. This implies that by simply modulating the concentration of GO in ink, we can fine-tune the layer formation while preventing the 
formation of aggregates, as the NC whiskers are spaced out. The NC whiskers are practically trapped in an electrostatic cage by the repulsive long-range forces exerted by GO sheets, as also observed for other similar charged colloidal rods ${ }^{45,46}$. As a result, the NC whiskers are embedded within the morphological order, leading to programmed directionality across the GO plane, otherwise unattainable in pure NC films. In particular, in the presence of discoid sheets with a dipole perpendicular to the sheet (in essence, GO), different sheets would prefer to order themselves along the dipolar direction predominantly. Having stiff NC whiskers in the dispersion, however, leads to the breaking of mirror symmetry, transforming the structure into a helical morphology within which the local polarisation alternates with the LC director on the $Z_{\text {nano }}$ direction.

Atomic force microscopy (AFM) investigation of the 3D printed structures (the very top Xnano- Ynano plane) shows the finest layered structure is accomplished in the sample with the highest amount of GO (Figure. 6H). This result is in agreement with the XRD data that showed a decrease in the $d$-spacing as the GO fraction increases. Analyzing the directionality histograms reveals the highest unidirectionality of NC whiskers is achieved in the samples containing up to 5\% GO. The improved orientation agrees with our synchrotron-based small-angle X-ray scattering measurements, which confirm a higher fraction of the oriented LC phase in samples containing higher amounts of GO.

Nevertheless, higher GO fraction (10\%) seems to strengthens the helical orientation of the NC whiskers. This might be due to higher viscosity (see the next section) of the ink containing a higher fraction of GO $(10 \%$ vs. $5 \%)$ that prevents them from being oriented toward the shear field and maintain the original helical orientation. This resulted in the architecture to become degenerated to handedness consisting of right- and left-handed helix. Increasing the amount of GO puts the sheets in a long row along the dipole, giving us the ability to control and fine-tune the ordering at the nanoscale.

\section{Rheological investigation}

A critical aspect of successful integration of the LC order into the $3 \mathrm{D}$ printed structure is the rheological properties of the ink ${ }^{47}$. To study the dynamics of the as-prepared inks and investigate the ease at which our LC-ink can flow, shape, and maintain the intrinsic order as stiffen into the desired form, we performed rheological studies. The low viscosity of the ink results in the formation of satellite droplets. The satellite parts of the 
drop, which usually go unnoticed, contribute to unaccounted errors and lower printability ${ }^{48,49}$. Moreover, low viscosity often causes the ink to spread on the surface, leading to low resolution and, consequently, difficulty at printing continuous lines. Low viscosity can also lead to the leakage of the liquid out of the nozzle.
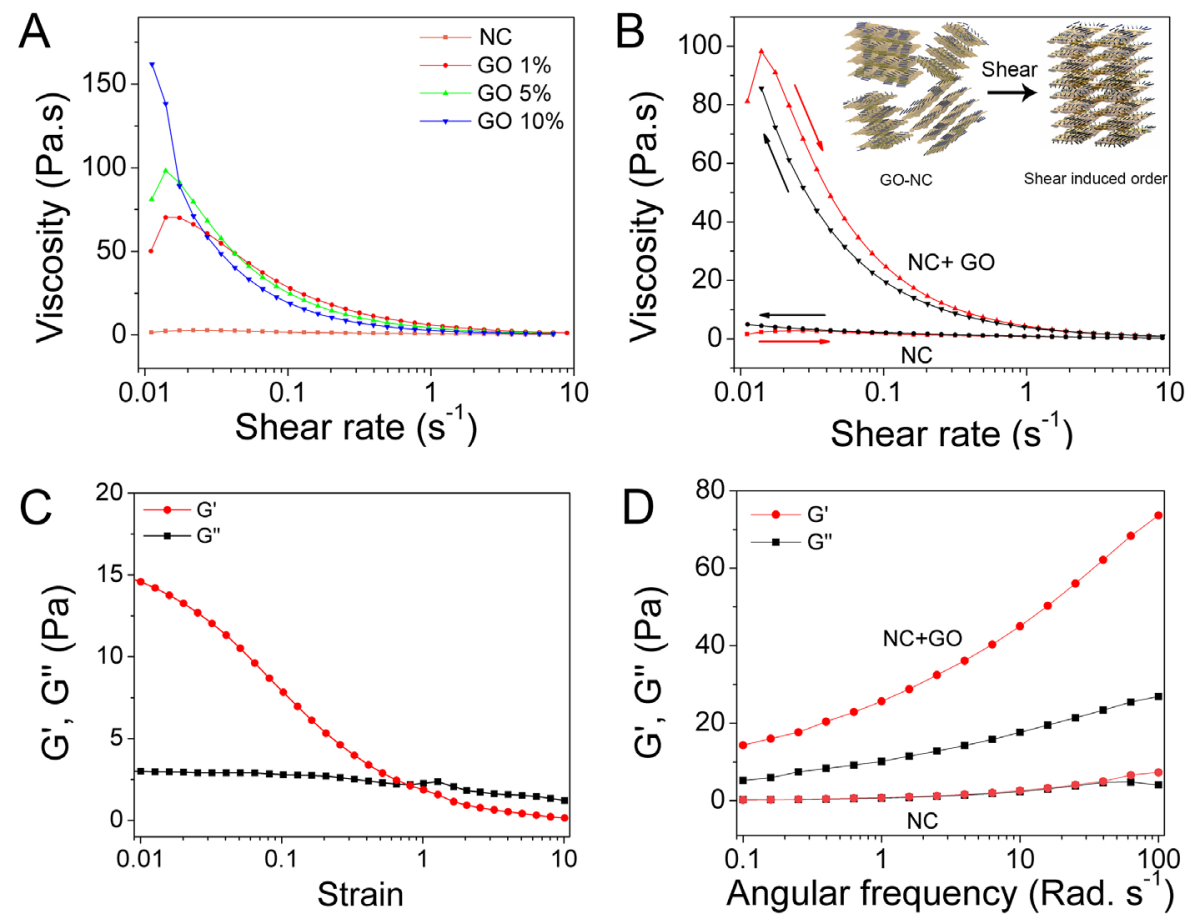

Figure. 7. Rheological investigation sheds light on the flow behaviour and the ability of our inks to maintain their intrinsic orientation after printing ( $\phi_{\text {solid }}=1.8 \mathrm{vol} \%$ for all samples). In contrast to NC whiskers, which show a negligible zero shear viscosity, even the addition of small amounts of GO $\left(\phi_{\mathrm{GO}}=0.018 \mathrm{vol} \%\right)$ resulted in a significant increase in viscosity $(\mathbf{A})$. This value is even higher than the onset of graphene oxide viscoelastic gels (50 vs. 3.8 Pa.s), suggesting easy printing of the as-prepared binary systems. (B) comparison of the viscosity vs. shear rate of the $\mathrm{NC}$ dispersion with a typical composite ( $5 \mathrm{vol} \% \mathrm{GO} / \mathrm{NC}$ ) showing the first two complete ascending-descending flow curves in logarithmic scale (arrows represent the direction of flow curve measurement). Inset, a schematic illustration of the shear-induced orientation process. (C) Storage $\left(G^{\prime}\right)$ and loss moduli $\left(G^{\prime \prime}\right)$ as a function of strain $(\mathbf{C}$, frequency of $0.1 \mathrm{~Hz})$ and frequency ( $\mathbf{D}$, the strain of 0.1 ). This is characterized by a weak non-universal exponent (a slow increase of storage modulus upon increasing the frequency), as depicted in (D). This suggests the formation of a robust ink at all timescales. 
Therefore, the ink should exhibit sufficiently high viscosity while maintaining low surface tension to prevent droplet agglomeration while at the same time allowing for near-complete wetting of the surface. Graphene and graphene oxide can serve as processing aids in this regard ${ }^{50,51}$. While the addition of GO results in the dynamic arrest $^{52}$ of the NC whiskers, GO, as an amphiphilic material ${ }^{53}$, can reduce the surface tension substantially improving the printability ${ }^{51}$.

The zero shear viscosity of the NC dispersion was measured to be $1.6 \mathrm{~Pa}$.s while the addition of $0.018,0.09$ and 0.18 vol.\% GO (corresponding to 1.28, 2.7 and 3.5 Pa.s ) to the NC dispersion resulted in a sharp increase to 50 Pa.s, 81 Pa.s and 162 Pa.s, respectively (Figure. 7A). The viscosity of composite dispersions is within the previously suggested range for GO-based $3 \mathrm{D}$ printing inks ${ }^{51}$. Such a strong synergistic effect can be attributed to the existence of a collective response of the components due to the self-rearrangement of GO sheets in the direction of the force field.

The sudden increase in the viscosity also suggests the formation of a network system resembling the rheological behaviour of soft glassy materials or cells ${ }^{54}$. Moreover, the flow curves of the composite dispersions showed yield values (the lowest shear stress necessary to produce viscous flow), typical behaviour of non-Newtonian fluids ${ }^{55}$. We previously showed that finite yield stress in the GO dispersions, containing ultra-large sheets, could occur at very low volume fractions of approximately $\varnothing>2.2 \times 10^{-4} 56$. Please note, this partial volume fraction of $\mathrm{GO}$ is too low $(\phi \mathrm{GO}=0.018 \mathrm{Vol} \%)$ to produce a viscous flow. Over the application of shear, the shear force is transferred from large $\mathrm{GO}$ sheets to small $\mathrm{NC}$ whiskers resulting in a collective viscous response. As the fraction of GO increases, so does the order of the layered LC phase, much more efficient propagation of the shear force within the network results in much higher viscosities.

To further illustrate the collective response of the components, a set of flow tests performed when the shear rate increased from $0.01 \mathrm{~s}^{-1}$ to $10 \mathrm{~s}^{-1}$ then slowed back down to $0.01 \mathrm{~s}^{-1}$. The corresponding ascending and descending curves presented in Figure. 7B. In both samples, the viscosity on the ascending curve did not match that of the descending curve. Interestingly, in the case of NC whiskers, the viscosity increased as opposed to the ascending curve. This increase in the viscosity is due to tumbling, randomization, agglomeration or bundling of $\mathrm{NC}$ whiskers that results in increased jamming among the particles. On the other hand, when the dispersion containing GO is subject to the shear 
field, the network of NC whiskers that is sandwiched between the ordered GO sheets flow more easily. This confirms that a shear-induced orientation takes place when they are subject to shear stress ${ }^{42,56-58}$. This behaviour suggests the possibility of a network structure, which is the direct result of the dynamic association of the NC whiskers in between the GO sheets, guiding the flow behaviour and is consistent with the observation of yield stress at zero shear.

The rheological modification can be further verified upon performing strain sweep at low frequencies on the binary inks Figure. 7C. The strain sweep was performed at low frequencies as not to disturb the internal structure, keeping the as-formed system intact and undisturbed. At small strains, the system experiences the overall dominance of $\mathrm{G}^{\prime}$ over G" (G' being around one order of magnitude higher than $\left.G^{\prime \prime}\right)$, implying the gelation of the layered structure as a result of network formation, resembling a viscoelastic soft solid. This quality indicates that there is enough time for the $\mathrm{NC}$ whiskers to re-orient themselves corresponding to a gel-like glassy state. However, this behaviour, in fact, different from the kinetic arrest observed in the case of rod shape materials such as single-walled carbon nanotubes ${ }^{59}$, which prevents them from the formation of the LC phase or aggregation over the extrusion process.

Figure. $7 \mathrm{D}$ shows the frequency sweep in a constant strain. In contrast to the $\mathrm{NC}$ whiskers sample, the storage modulus was dominant in the composite sample. This suggests that upon the phase modification, a semi-permanent gel-like phase (network) formed. Quite interestingly, even at very short timescales, no cross-over point could be observed, suggesting the robustness of the as-prepared gel phase. Therefore, the printed structure that is finally obtained after drying, in principle, resembles the structure before drying, allowing us to maintain the original LC order at the nanoscale in the final asdried printed 3D microarchitecture.

\section{Printed relative humidity sensors}

Using the developed method, we can print unlimited lengths and various shapes of 3D constructs without losing the original order. This allows manufacturing of highperformance functional devices in which controlling the spacing and orientation within the nanoscale is of utmost importance, such as relative humidity $(\mathrm{RH})$ sensors ${ }^{60,61}$. As a proof of concept, we employed the liquid crystal mediated printing to fabricate $\mathrm{RH}$ 
sensors constituting self-assembled lamellar organization of NC whiskers. The NC whiskers were printed as the active $\mathrm{RH}$ sensing material on quartz crystal microbalance (QCM) transducers (Figure. $8 \mathrm{~A}$ ). The printing strategy was a very powerful tool for us to deliver a controlled amount of highly ordered LC-ink that was not possible by hand drop-casting.
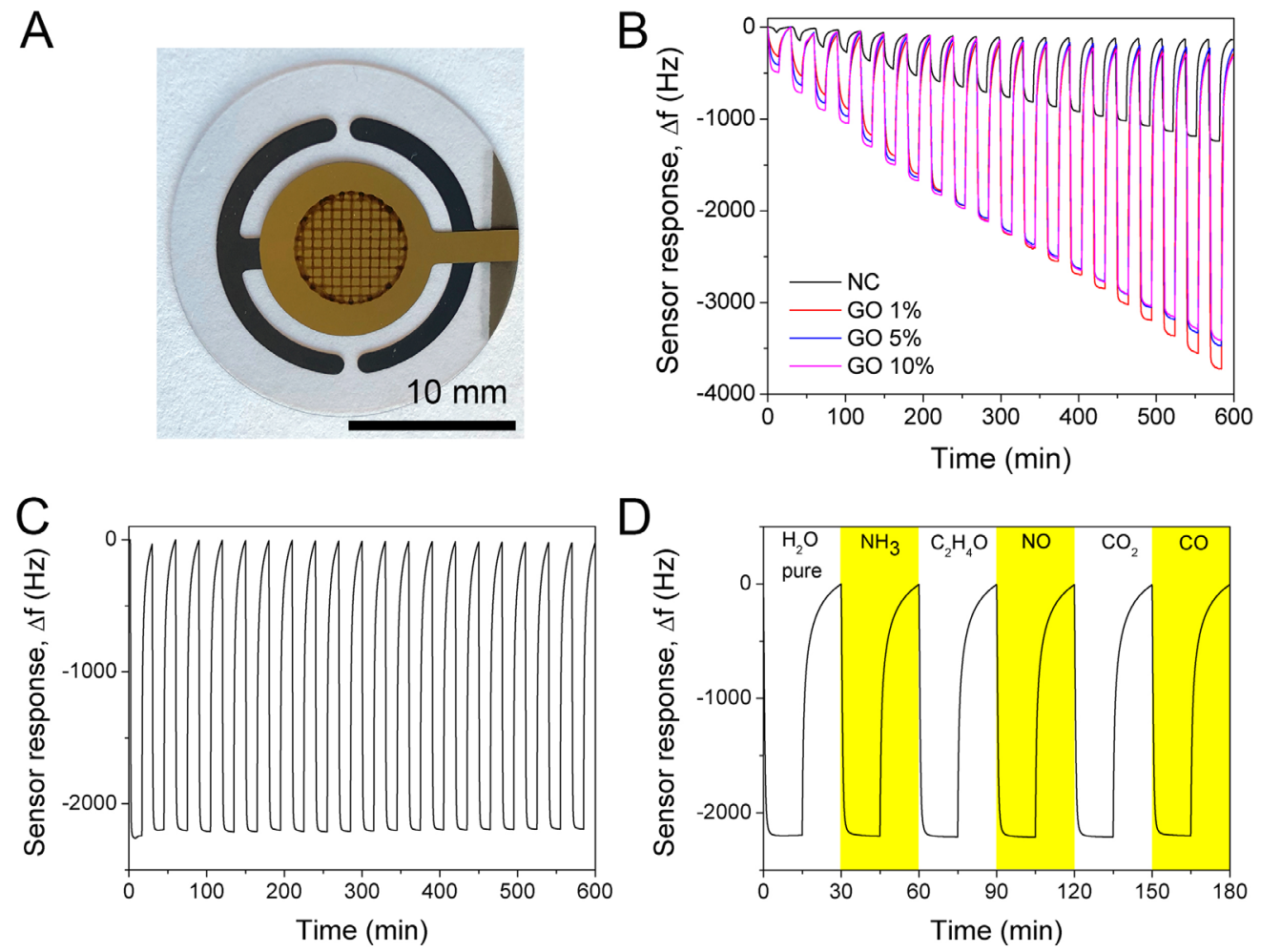

Figure. 8. Humidity sensor production. (A) A representative image of a liquid crystal mediated 3D printed mesh on top of the QCM electrode to fabricate the humidity sensor. (B) The humidity uptake test where the sensors were exposed to 20 different concentrations of humidity ranging from 0.6 to $23 \% \mathrm{RH}$ at $27^{\circ} \mathrm{C}$. (C) Repeatability performance of the humidity sensors at a fixed humidity of $13 \%$ RH. (D) Selectivity test of the NC- based HR sensors toward humidity in the presence of various industrial relevant gas species. The dynamic response of the QCM electrode is not affected as a result of the contaminant gases.

The pure NC whiskers-based humidity sensor revealed a relatively weak dynamic response (sensitivity). In contrast, the sensor with a programmed directionality of the active materials showed a significantly higher sensitivity $(420 \mathrm{~Hz} / \% \mathrm{RH})$ with excellent reproducibility (Figure. 8 B-C). Please note, even though, GO (a hydrophilic material) 
can similarly contribute to the absorption of the moisture, the most significant improvement in the performance was observed by the addition of $1 \%$ GO. The sensitivity of the sensor is over one order of magnitude better than the highest ever reported sensitivities of similar systems, such as $28.7 \mathrm{~Hz} / \% \mathrm{RH}$ for the copper metalorganic framework and $66.5 \mathrm{~Hz} / \% \mathrm{RH}$ for pure GO as the sensitive layer ${ }^{60,62}$. Such a high sensitivity results in an exceptionally large signal-to-noise ratio $(\sim 2200 \mathrm{~Hz} / \mathrm{Hz})$, thus allowing for trace levels of humidity to be detected. The sensor displayed an extremely low limit of detection (LOD) of $1 \mathrm{ppm}$ absolute humidity, a level that has never been reported to date. This LOD is at least one order of magnitude superior to the best-performing materials reported in the literature, including and not limited to $2 \mathrm{D}$ MXenes, gold, graphene, and copper ${ }^{63}$.

Although the hydrophilic functional groups on the surface of $\mathrm{NC}$ whiskers provide a high potential to adsorb water molecules, the densely packed NC film does not support an easy moisture uptake. Conversely, the $\mathrm{NC}$ whiskers that are flat and parallel to each other in the laminar organization allow facile mass transport through the structure. This effect can be further demonstrated by calculating the sorption rate constant kads of the first-order water transfer reaction ${ }^{64}$. The $k a d s$ increases from $0.0136 \mathrm{~s}^{-1}$ to $0.0316 \mathrm{~s}^{-1}$ when the NC self-assemble from randomly agglomerated whiskers to the laminar organization. This superb performance is attributed to the much higher available surface area when numerous channels are created in each printed layer.

The NC sensor was also found to possess excellent selectivity, which puts it at the forefront of the best-performing humidity sensors reported in the literature (Figure. 8D). The sensors' detection range is shown to be from trace levels to up to $>20 \% \mathrm{RH}$ with unparalleled accuracy and selectivity. As such, we fabricated ultra-responsive humidity sensors with intricate geometries from the feedstock in one single step that outperforms any commodity or advanced materials in terms of performance. Therefore, the 3D printed RH sensor developed here is one of the first devices of its kind to exhibit all of the required traits of a good humidity sensor namely, production cost, sensitivity, repeatability, response time, wide concentration detection range and selectivity performances. The device can, therefore, have a wide range of applications and be safely used in monitoring the environment, human/animal respiration, food quality, indoor air 
quality, chemical industry processing, and trace moisture in high-purity gases in the semiconductor industry.

\section{Conclusions}

Here by exploring anisotropic properties of one-dimensional stiff NC whiskers and super-flexible two-dimensional giant GO building blocks, we created a 3D printable ink that was used for demonstrating the conceptual multi-scale printing process. The process combined the shaping freedom of additive manufacturing with nano-level ordering. The introduced directed self-assembly approach appeared to be an empowering tool to exploit the nano-assembly within 3D printing of mechanically robust architectures. Because large GO sheets can support similar LC phases in a wide range of organic solvents ${ }^{14}, 3 \mathrm{D}$ printing of layer-by-layer 3D structures containing ordered nanomaterials that are not processable in water is also possible. The ease of synthesis, much shorter processing time and high scalability of this route offer facile fabrication of $3 \mathrm{D}$ frameworks with valuable properties.

These architectures are suitable for industrial applications, as demonstrated for the HR sensor. That is the merger of an already established application in which a deep fundamental understanding exists with an up-and-coming technology that enables the scale-up of complex structures to develop and discover innovative processes and systems. This methodology can be adopted for many more applications such as electrocatalysis, supercapacitors and batteries that require scaffolding with $3 \mathrm{D}$ conductive substrates, fine-tuning micro and macropore channels, configuring hierarchical structures, aligning the internal constructions via a self-assembly process to optimize the electrochemical properties of the active materials in the final device.

With this development, NCs has been shown as both reinforcing filler in green composites and also as the main building block with many remarkable characteristics. This modification leads to true freedom of design in conventional direct ink writing methods. Hence, this route can serve as a source for the development and growth of sustainable and green multi-scale printing strategies.

\section{Materials and Methods}


GO was synthesized with a method described previously ${ }^{65}$. Briefly, natural graphite powder $(1 \mathrm{~g})$ and sulphuric acid $(200 \mathrm{~mL})$ were mixed and stirred in a flask for one $\mathrm{h}$. Then $\mathrm{KMnO}_{4}(10 \mathrm{~g})$ was added to the mixture and stirred for one day. The mixture transferred into an ice bath, and Milli-Q (200 mL) water was added slowly before $\mathrm{H}_{2} \mathrm{O}_{2}$ $(50 \mathrm{~mL})$ poured into the mixture. Having stirred for another $30 \mathrm{~min}$, the GO particles then washed and centrifuged three times with $\mathrm{HCl}$ solution ( $9: 1$ water/ $\mathrm{HCl}$ by volume), then centrifuged again and washed with Milli-Q water until the $\mathrm{pH}$ of the solution became about 4-5. Nanocellulose was synthesized with a method described previously ${ }^{66}$. Bleached softwood kraft pulp was used as the cellulose material. Ten grams of dried pulp was treated with $100 \mathrm{ml}$ of $65 \%$ (w/w) $\mathrm{H}_{2} \mathrm{SO}_{4}$ at $70^{\circ} \mathrm{C}$ for $10 \mathrm{~min}$ with continuous stirring. The sample was then repeatedly washed with centrifugation $\left(1600 \mathrm{~g}\right.$, at $10^{\circ} \mathrm{C}$ for $5 \mathrm{~min}$ ). When the $\mathrm{pH}$ of the sample reached $1-3$, the fine particle component did not sediment, and the supernatant became turbid. The turbid supernatant was collected and thoroughly dialyzed against distilled water. The suspension was finally sonicated with a rod-type sonicator for $1 \mathrm{~min}$.

In the case of the mixtures, 120 samples were prepared following the table S2. All the mixtures and 60 other samples of GO $(0.0125 \mathrm{mg} / \mathrm{ml}$ up to $15 \mathrm{mg} / \mathrm{ml})$ and $\mathrm{NC}(1 \mathrm{mg} / \mathrm{ml}$ to $17 \mathrm{mg} / \mathrm{ml}$ ) were prepared and tested for Small-angle X-ray scattering (SAXS) beamline at the Australian Synchrotron, Clayton, Australia. Samples were loaded into $1.5 \mathrm{~mm}$ individual glass capillaries and sealed with beeswax to prevent water absorption or solvent loss. Specimens were housed in a 48-sample capillary holder, with the temperaturecontrolled using a Huber recirculating water bath. Scattering patterns were acquired for all samples at $25^{\circ} \mathrm{C}$. Utilizing a camera length of $7 \mathrm{~m}$, the obtained SAXS $q$ range was from 0.0015 to $0.2 \AA^{-1}$. The contribution from an empty capillary and water was subtracted from the scattering profiles. The 2D patterns were converted to the SAXS spectra and were modelled using SasView software (http://www.sasview.org/).

Rheological properties of various concentrations of GO dispersion were investigated using a TA rheometer (AR G2). A cone-plate geometry $\left(60 \mathrm{~mm}, 2^{\circ}\right)$ was used to perform experiments. Different volume ratios of $\mathrm{NC} / \mathrm{GO}$ were mixed thoroughly and then allowed to rest overnight before each rheological test. After loading the rheometer with samples $(2.1 \mathrm{ml})$ with various $\mathrm{NC} / \mathrm{GO}$ ratio, samples were allowed to rest in the final position for another $4 \mathrm{hrs}$ at the experimental temperature $\left(25^{\circ} \mathrm{C}\right)$, to eliminate any 
viscoelastic history. No prestressing condition was applied to prevent ordering in the samples. A solvent trap filled with distilled water was used to avoid samples of losing water throughout long experiments. Zero shear viscosity measured at a very low shear rate of $0.01 \mathrm{~s}^{-1}$ to avoid the dispersion produce any shear stress. The relaxation dynamics of the dispersions were studied by measuring the storage modulus, and loss modulus of the dispersions with various $\mathrm{NC} / \mathrm{GO}$ concentration as a function of frequency at a constant strain amplitude of 0.01 . This strain amplitude was chosen to prevent samples of going under large deformations. In the preliminary shear experiments, it was shown that strains larger than 0.01 could result in a structural change. Consequently, the larger deformations could mask the relaxation behaviour of the dispersions. The zero-shear viscosity was measured using a simple shear experiment in which shear rate was ramped from $10^{-2} \mathrm{~s}^{-1}$ up to $10 \mathrm{~s}^{-1}$ then ramped back to $10^{-2} \mathrm{~s}^{-1}$.

3D printing experiments were carried out using a Hyrel 3D printer (Engine High Resolution), USA. The extrusion rate of the materials was controlled depending on the needle and material concentration. Printing parameters used were as follow: speed: 15 $\mathrm{mm} / \mathrm{sec}$ to $100 \mathrm{~mm} / \mathrm{s}$, nozzle: 32 gage, $\phi_{\mathrm{NC}}=1.8 \mathrm{vol} \%, \phi_{\mathrm{GO}}=0.018 \mathrm{vol} \%$, GO $1 \mathrm{vol} \% \mathrm{vs} \mathrm{NC}$.

The morphology and cross-section of printed structures were imaged by a Nova Nano SEM 450 and high-resolution TEM (Phillips CM200, Amsterdam, Netherlands). Directionality histograms were computed using Fiji, ImageJ. FIB sample processing was performed on a Scios Dual Beam Thermo Fisher FEI FIB/SEM. Sections were cut from a one-layer printed film on a SI wafer substrate. A cross-sectional cut was made into the surface to reveal the cross-section of the layered GO-NC material. A 7nA $\mathrm{Ga}+$ ion current was used for the initial cross-sectional cut then the exposed crosssection was ion beam polished using a $100 \mathrm{pA}$ current.

The quartz crystal microbalance (QCM) devices were fabricated according to our recently reported process ${ }^{60}$. Briefly, the QCMs used had an oscillation frequency of $\sim 10 \mathrm{MHz}$. The QCM had Ti electrodes $(\varnothing=4.5 \mathrm{~mm}$ and $300 \mathrm{~nm}$ thickness), which were deposited via e-beam evaporation on each side of the quartz substrate. The deposited mass of NC whiskers-based materials was determined using the QCM data from an Agilent E5100A network. The process involved NC whiskers dispersions from different mixes being $3 \mathrm{D}$ printed onto the QCM device and dried at room temperature. The centre frequency changes of the QCMs were monitored using a Research Quartz Crystal 
Microbalance (RQCM, Maxtek) frequency counter, which had a frequency resolution of $\pm 0.03 \mathrm{~Hz}$.

The humidity uptake measurements were performed at a constant operating temperature of $27^{\circ} \mathrm{C}$ and a total gas flow rate of $200 \mathrm{~mL} / \mathrm{min}$ throughout the experiments. A multichannel gas delivery system with computer-controlled mass flow controllers (MKS Instruments, Inc. USA) was used to deliver the gas to the sensor chamber. The different humidity levels (0.6 to $23 \% \mathrm{RH}$ ) were controlled through a V-Gen (from InstruQuest) humidity generator. The humidity sensing experiments involved exposing the sensors to humidity/nitrogen atmospheres for 15minutes / 15 minutes sequences. Each 15/15 min exposure/recovery sequence is referred to as a "pulse". The selectivity and repeatability experiments were performed with a $13 \% \mathrm{RH}$ level at $27^{\circ} \mathrm{C}$. Selectivity tests involved the exposure to ammonia $\left(\mathrm{NH}_{3}\right)$, acetaldehyde $\left(\mathrm{C}_{2} \mathrm{H}_{4} \mathrm{O}\right)$, nitric oxide $(\mathrm{NO})$, carbon dioxide $\left(\mathrm{CO}_{2}\right)$ and oxygen $\left(\mathrm{O}_{2}\right)$ with/without the presence of humidity $(13 \% \mathrm{RH}$ level at $\left.27^{\circ} \mathrm{C}\right)$.

The sorption rate constant $k_{a d s}$ was calculated by the first-order water transfer reaction following equation $1^{64}$.

$\ln \left(\frac{M_{\infty}-M_{0}}{M_{\infty}-M_{(t)}}\right)=k_{\text {sorption }} t$

Where $M_{\infty}$ is the final mass change of the NC whiskers film, $M_{0}$ is the mass change of the film at the initial time $(0 \mathrm{~g})$ upon exposure to $\mathrm{RH} 13 \%$ condition, and $M_{(t)}$ is the absorbed mass of $\mathrm{H}_{2} \mathrm{O}$ at time $t$.

\section{Conflicts of interest}

There are no conflicts to declare.

\section{Author contributions}

R.J. and D.E. conceptualized, directed the research project and wrote the manuscript. R.J has performed GO synthesis, XRD, AFM, POM, Rheological studies, mechanical tests and TEM sample preparation. 
SEM of the 3D printed samples has been performed by J.S and M.M.

The 3D Printings have been performed by R.J., D.E. and B.H.R.S.

Synchrotron SAXS studies have been performed by R.J., A.S. and D.E.

S.N., C.Z. and P.P. have contributed to interpretation of SAXS and LC data analysis.

M.B.G., A.S., E.M. and R.J. were responsible for the TEM analysis.

J.A. Synthesized NC whisker suspensions and contributed to the conceptualization of the LC mixed.

Y.S., A.E.K, and S.B. were in charge of HR sensor fabrication and tests.

R.A. provided support, guidance and proof-read the manuscript; all authors discussed the results and commented on the manuscript.

\section{Acknowledgements}

R.J. acknowledges the Australian Research Council (ARC) DECRA Fellowship grant (DE180100215). D.E. acknowledges the Scientia Fellowship scheme at The University of New South Wales (USNW). The authors are grateful to Australian Synchrotron for beam time awards to this project and the staff on the SAXS/WAXS beamline at the Australian Synchrotron, part of ANSTO.

\section{Supporting information}

The supporting discussions section includes following sections. Section 1: Small-angle X-ray scattering study of NC and GO (Figure S1-5). Section 2: Processability of NC whiskers (Figure S6). Section 3: Templating the NC mesogens (Figure S7). Section 4: Layered structural order of the binary system (Figure S8). Section 5: Flow alignment behavior of the binary system (Figure S9). Section 6: Robustness of the binary system (Figure S10-11). Section 7: Optimizing 3D printing process (Figure S12-14). Section 8: Z-resolutions (Figure S15). Section 9: The d-spacing as a function of GO fraction (Figure S16). Section 10: Mechanical performance of the printed films (Table S1). Section 11: Synchrotron sample information (Table S2). Movies S1-2 and supporting references. 


\section{References}

1. Capel, A. J.; Rimington, R. P.; Lewis, M. P.; Christie, S. D. R., 3D printing for chemical, pharmaceutical and biological applications. Nat. Rev. Chem. 2018, 2 (12), 422-436.

2. Ambrosi, A.; Pumera, M., 3D-printing technologies for electrochemical applications. Chem. Soc. Rev. 2016, 45 (10), 2740-2755.

3. Friedmann, D.; Lee, A. F.; Wilson, K.; Jalili, R.; Caruso, R. A., Printing approaches to inorganic semiconductor photocatalyst fabrication. J. Mater. Chem. A 2019, 7 (18), 1085810878.

4. El-Sayegh, S.; Romdhane, L.; Manjikian, S., A critical review of 3D printing in construction: benefits, challenges, and risks. Arch. Civ. Mech. Eng. 2020, 20 (2), 34.

5. Ngo, T. D.; Kashani, A.; Imbalzano, G.; Nguyen, K. T. Q.; Hui, D., Additive manufacturing (3D printing): A review of materials, methods, applications and challenges. Compos. B. Eng 2018, 143, 172-196.

6. Elder, B.; Neupane, R.; Tokita, E.; Ghosh, U.; Hales, S.; Kong, Y. L., Nanomaterial Patterning in 3D Printing. Adv. Mater. 2020, 32 (17), 1907142.

7. Zhang, Q.; Zhang, F.; Medarametla, S. P.; Li, H.; Zhou, C.; Lin, D., 3D Printing of Graphene Aerogels. Small 2016, 12 (13), 1702-1708.

8. Gantenbein, S.; Masania, K.; Woigk, W.; Sesseg, J. P. W.; Tervoort, T. A.; Studart, A. R., Three-dimensional printing of hierarchical liquid-crystal-polymer structures. Nature 2018, 561 (7722), 226-230.

9. Ambulo, C. P.; Burroughs, J. J.; Boothby, J. M.; Kim, H.; Shankar, M. R.; Ware, T. H., Four-dimensional Printing of Liquid Crystal Elastomers. ACS Applied Materials \& Interfaces 2017, 9 (42), 37332-37339.

10. Laurent, J.; Blin, G.; Chatelain, F.; Vanneaux, V.; Fuchs, A.; Larghero, J.; Théry, M., Convergence of microengineering and cellular self-organization towards functional tissue manufacturing. Nat. Biomed. Eng. 2017, 1 (12), 939-956.

11. Glotzer, S. C.; Solomon, M. J., Anisotropy of building blocks and their assembly into complex structures. Nat. Mater. 2007, 6, 557.

12. Sasai, Y., Cytosystems dynamics in self-organization of tissue architecture. Nature 2013, 493, 318.

13. Dierking, I.; Al-Zangana, S., Lyotropic Liquid Crystal Phases from Anisotropic Nanomaterials. Nanomaterials 2017, 7 (10), 305.

14. Jalili, R.; Aboutalebi, S. H.; Esrafilzadeh, D.; Konstantinov, K.; Moulton, S. E.; Razal, J. M.; Wallace, G. G., Organic Solvent-Based Graphene Oxide Liquid Crystals: A Facile Route toward the Next Generation of Self-Assembled Layer-by-Layer Multifunctional 3D Architectures. ACS Nano 2013, 7 (5), 3981-3990.

15. Wang, K.; Frewin, C. L.; Esrafilzadeh, D.; Yu, C.; Wang, C.; Pancrazio, J. J.; RomeroOrtega, M.; Jalili, R.; Wallace, G., High-Performance Graphene-Fiber-Based Neural Recording Microelectrodes. Adv. Mater. 2019, 31 (15), 1805867.

16. Park, K. S.; Kwok, J. J.; Dilmurat, R.; Qu, G.; Kafle, P.; Luo, X.; Jung, S.-H.; Olivier, Y.; Lee, J.-K.; Mei, J.; Beljonne, D.; Diao, Y., Tuning conformation, assembly, and charge transport properties of conjugated polymers by printing flow. Sci. Adv. 2019, 5 (8), eaaw7757.

17. Liu, Y.; Xu, Z.; Gao, W.; Cheng, Z.; Gao, C., Graphene and Other 2D Colloids: Liquid Crystals and Macroscopic Fibers. Adv. Mater. 2017, 29 (14), 1606794. 
18. Schirmer, K. S. U.; Esrafilzadeh, D.; Thompson, B. C.; Quigley, A. F.; Kapsa, R. M. I.; Wallace, G. G., Conductive composite fibres from reduced graphene oxide and polypyrrole nanoparticles. J. Mater. Chem. B 2016, 4 (6), 1142-1149.

19. Kotikian, A.; Truby, R. L.; Boley, J. W.; White, T. J.; Lewis, J. A., 3D Printing of Liquid Crystal Elastomeric Actuators with Spatially Programed Nematic Order. Adv. Mater. 2018, 30 (10), 1706164.

20. Hausmann, M. K.; Rühs, P. A.; Siqueira, G.; Läuger, J.; Libanori, R.; Zimmermann, T.; Studart, A. R., Dynamics of Cellulose Nanocrystal Alignment during 3D Printing. ACS Nano 2018, 12 (7), 6926-6937.

21. Athukoralalage, S. S.; Balu, R.; Dutta, N. K.; Choudhury, N. R., 3D Bioprinted Nanocellulose-Based Hydrogels for Tissue Engineering Applications: A Brief Review. Polymers 2019, 11, 898.

22. Wang, X.; Wang, Q.; Xu, C., Nanocellulose-Based Inks for 3D Bioprinting: Key Aspects in Research Development and Challenging Perspectives in Applications-A Mini Review. Bioengineering 2020, 7 (2), 40.

23. Xu, C.; Zhang Molino, B.; Wang, X.; Cheng, F.; Xu, W.; Molino, P.; Bacher, M.; Su, D.; Rosenau, T.; Willför, S.; Wallace, G., 3D printing of nanocellulose hydrogel scaffolds with tunable mechanical strength towards wound healing application. J. Mater. Chem. B 2018, 6 (43), 7066-7075.

24. Håkansson, K. M. O.; Henriksson, I. C.; de la Peña Vázquez, C.; Kuzmenko, V.; Markstedt, K.; Enoksson, P.; Gatenholm, P., Solidification of 3D Printed Nanofibril Hydrogels into Functional 3D Cellulose Structures. Adv. Mater. Technol. 2016, 1 (7), 1600096.

25. Cataldi, A.; Rigotti, D.; Nguyen, V. D. H.; Pegoretti, A., Polyvinyl alcohol reinforced with crystalline nanocellulose for 3D printing application. Mater. Today Commun. 2018, 15, 236-244.

26. Pan, J.; Hamad, W.; Straus, S. K., Parameters Affecting the Chiral Nematic Phase of Nanocrystalline Cellulose Films. Macromolecules 2010, 43 (8), 3851-3858.

27. Poulin, P., How to achieve a successful biaxial marriage. Science 2018, 360 (6390), 712-713.

28. Araki, J.; Kuga, S., Effect of Trace Electrolyte on Liquid Crystal Type of Cellulose Microcrystals. Langmuir 2001, 17 (15), 4493-4496.

29. Klemm, D.; Cranston, E. D.; Fischer, D.; Gama, M.; Kedzior, S. A.; Kralisch, D.; Kramer, F.; Kondo, T.; Lindström, T.; Nietzsche, S.; Petzold-Welcke, K.; Rauchfuß, F., Nanocellulose as a natural source for groundbreaking applications in materials science: Today's state. Mater. Today 2018, 21 (7), 720-748.

30. Abitbol, T.; Rivkin, A.; Cao, Y.; Nevo, Y.; Abraham, E.; Ben-Shalom, T.; Lapidot, S.; Shoseyov, O., Nanocellulose, a tiny fiber with huge applications. Curr. Opin. Biotechnol. 2016, 39, 76-88.

31. Lee, K.-Y.; Aitomäki, Y.; Berglund, L. A.; Oksman, K.; Bismarck, A., On the use of nanocellulose as reinforcement in polymer matrix composites. Compos. Sci.Technol. 2014, 105, 15-27.

32. Araki, J.; Wada, M.; Kuga, S.; Okano, T., Birefringent Glassy Phase of a Cellulose Microcrystal Suspension. Langmuir 2000, 16 (6), 2413-2415.

33. Lagerwall, J. P. F.; Schütz, C.; Salajkova, M.; Noh, J.; Hyun Park, J.; Scalia, G.; Bergström, L., Cellulose nanocrystal-based materials: from liquid crystal self-assembly and glass formation to multifunctional thin films. NPG Asia Mater. 2014, 6, e80. 
34. Jalili, R.; Aboutalebi, S. H.; Esrafilzadeh, D.; Shepherd, R. L.; Chen, J.; AminorroayaYamini, S.; Konstantinov, K.; Minett, A. I.; Razal, J. M.; Wallace, G. G., Scalable One-Step Wet-Spinning of Graphene Fibers and Yarns from Liquid Crystalline Dispersions of Graphene Oxide: Towards Multifunctional Textiles. Adv. Funct. Mater. 2013, 23 (43), 5345-5354.

35. Mao, Y.; Liu, K.; Zhan, C.; Geng, L.; Chu, B.; Hsiao, B. S., Characterization of Nanocellulose Using Small-Angle Neutron, X-ray, and Dynamic Light Scattering Techniques. J. Phys. Chem. B 2017, 121 (6), 1340-1351.

36. Bai, L.; Huan, S.; Zhao, B.; Zhu, Y.; Esquena, J.; Chen, F.; Gao, G.; Zussman, E.; Chu, G.; Rojas, O. J., All-Aqueous Liquid Crystal Nanocellulose Emulsions with Permeable Interfacial Assembly. ACS Nano 2020, 14 (10), 13380-13390.

37. Colloidal Systems. In Biaxial Nematic Liquid Crystals, pp 305-318.

38. van der Kooij, F. M.; Lekkerkerker, H. N. W., Liquid-Crystal Phases Formed in Mixed Suspensions of Rod- and Platelike Colloids. Langmuir 2000, 16 (26), 10144-10149.

39. Woolston, P.; van Duijneveldt, J. S., Three-Phase Coexistence in Colloidal Rod-Plate Mixtures. Langmuir 2015, 31 (34), 9290-9295.

40. Vroege, G. J., Biaxial phases in mineral liquid crystals. Liquid Crystals 2014, 41 (3), 342-352.

41. Peroukidis, S. D.; Vanakaras, A. G.; Photinos, D. J., Liquid crystalline phases and demixing in binary mixtures of shape-anisometric colloids. J. Mater. Chem. 2010, 20 (46), 10495-10502.

42. Naficy, S.; Jalili, R.; Aboutalebi, S. H.; Gorkin lii, R. A.; Konstantinov, K.; Innis, P. C.; Spinks, G. M.; Poulin, P.; Wallace, G. G., Graphene oxide dispersions: tuning rheology to enable fabrication. Mater. Horiz. 2014, 1 (3), 326-331.

43. Schirmer, K. S. U.; Esrafilzadeh, D.; Thompson, B. C.; Quigley, A. F.; Kapsa, R. M. I.; Wallace, G. G., Conductive composite fibres from reduced graphene oxide and polypyrrole nanoparticles. Journal of Materials Chemistry B 2016, 4 (6), 1142-1149.

44. Román-Manso, B.; Figueiredo, F. M.; Achiaga, B.; Barea, R.; Pérez-Coll, D.; Morelos-Gómez, A.; Terrones, M.; Osendi, M. I.; Belmonte, M.; Miranzo, P., Electrically functional 3D-architectured graphene/SiC composites. Carbon 2016, 100, 318-328.

45. Kang, K.; Dhont, J. K. G., Structural arrest and texture dynamics in suspensions of charged colloidal rods. Soft Matter 2013, 9 (17), 4401-4411.

46. Mundoor, H.; Park, S.; Senyuk, B.; Wensink, H. H.; Smalyukh, I. I., Hybrid molecularcolloidal liquid crystals. Science 2018, 360 (6390), 768-771.

47. M'Barki, A.; Bocquet, L.; Stevenson, A., Linking Rheology and Printability for Dense and Strong Ceramics by Direct Ink Writing. Sci. Rep. 2017, 7 (1), 6017.

48. Wallevik, O. H.; Feys, D.; Wallevik, J. E.; Khayat, K. H., Avoiding inaccurate interpretations of rheological measurements for cement-based materials. Cement Concrete Res. 2015, 78, 100-109.

49. O'Mahony, C.; Haq, E. U.; Silien, C.; Tofail, S. A. M., Rheological Issues in CarbonBased Inks for Additive Manufacturing. Micromachines 2019, 10 (2), 99.

50. Valentini, L.; Bittolo Bon, S.; Fortunati, E.; Kenny, J. M., Preparation of transparent and conductive cellulose nanocrystals/graphene nanoplatelets films. J Mater. Sci. 2014, 49 (3), 1009-1013.

51. Corker, A.; Ng, H. C. H.; Poole, R. J.; García-Tuñón, E., 3D printing with 2D colloids: designing rheology protocols to predict 'printability' of soft-materials. Soft Matter 2019, 15 (6), 1444-1456. 
52. Perez, H.; Rojas-Ochoa, L.; Castaneda-Priego, R.; Quesada-Pérez, M.; CallejasFernandez, J.; Hidalgo-Alvarez, R.; Trappe, V., Dynamic Arrest in Charged Colloidal Systems Exhibiting Large-Scale Structural Heterogeneities. Phys. Rev. Lett 2009, 102, 018301.

53. Aboutalebi, S. H.; Aminorroaya-Yamini, S.; Nevirkovets, I.; Konstantinov, K.; Liu, H. K., Enhanced Hydrogen Storage in Graphene Oxide-MWCNTs Composite at Room Temperature. Adv. Energy Mater. 2012, 2 (12), 1439-1446.

54. Sollich, P.; Lequeux, F.; Hébraud, P.; Cates, M. E., Rheology of Soft Glassy Materials. Phys. Rev. Lett 1997, 78 (10), 2020-2023.

55. Zhu, H.; Kim, Y. D.; De Kee, D., Non-Newtonian fluids with a yield stress. J NonNewton Fluid 2005, 129 (3), 177-181.

56. Jalili, R.; Aboutalebi, S. H.; Esrafilzadeh, D.; Konstantinov, K.; Razal, J. M.; Moulton, S. E.; Wallace, G. G., Formation and processability of liquid crystalline dispersions of graphene oxide. Mater. Horiz. 2014, 1 (1), 87-91.

57. Jalili, R.; Aminorroaya-Yamini, S.; Benedetti, T. M.; Aboutalebi, S. H.; Chao, Y.; Wallace, G. G.; Officer, D. L., Processable 2D materials beyond graphene: MoS2 liquid crystals and fibres. Nanoscale 2016, 8 (38), 16862-16867.

58. Poulin, P.; Jalili, R.; Neri, W.; Nallet, F.; Divoux, T.; Colin, A.; Aboutalebi, S. H.; Wallace, G.; Zakri, C., Superflexibility of graphene oxide. Proc. Natl. Acad. Sci. 2016, 113 (40), 11088-11093.

59. Lagerwall, J. P. F.; Scalia, G., Carbon nanotubes in liquid crystals. J. Mater. Chem. 2008, 18 (25), 2890-2898.

60. Jalili, R.; Esrafilzadeh, D.; Aboutalebi, S. H.; Sabri, Y. M.; Kandjani, A. E.; Bhargava, S. K.; Della Gaspera, E.; Gengenbach, T. R.; Walker, A.; Chao, Y.; Wang, C.; Alimadadi, H.; Mitchell, D. R. G.; Officer, D. L.; MacFarlane, D. R.; Wallace, G. G., Silicon as a ubiquitous contaminant in graphene derivatives with significant impact on device performance. Nat. Commun. 2018, 9 (1), 5070.

61. Tulliani, J.-M.; Inserra, B.; Ziegler, D., Carbon-Based Materials for Humidity Sensing: A Short Review. Micromachines 2019, 10 (4), 232.

62. Zhou, Z.; Li, M.-X.; Wang, L.; He, X.; Chi, T.; Wang, Z.-X., Antiferromagnetic Copper(II) Metal-Organic Framework Based Quartz Crystal Microbalance Sensor for Humidity. Cryst. Growth Des. 2017, 17 (12), 6719-6724.

63. Sabri, Y. M.; Kandjani, A. E.; Ippolito, S. J.; Bhargava, S. K., Ordered Monolayer Gold Nano-urchin Structures and Their Size Induced Control for High Gas Sensing Performance. Sci. Rep. 2016, 6 (1), 24625.

64. Krtil, P.; Trojánek, A.; Samec, Z., Kinetics of Water Sorption in NafionThin Films Quartz Crystal Microbalance Study. J. Phys. Chem. B 2001, 105 (33), 7979-7983.

65. Aboutalebi, S. H.; Gudarzi, M. M.; Zheng, Q. B.; Kim, J.-K., Spontaneous Formation of Liquid Crystals in Ultralarge Graphene Oxide Dispersions. Adv. Funct. Mater. 2011, 21 (15), 2978-2988.

66. Araki, J.; Wada, M.; Kuga, S.; Okano, T., Flow properties of microcrystalline cellulose suspension prepared by acid treatment of native cellulose. Colloids Surf. A 1998, 142 (1), 7582. 


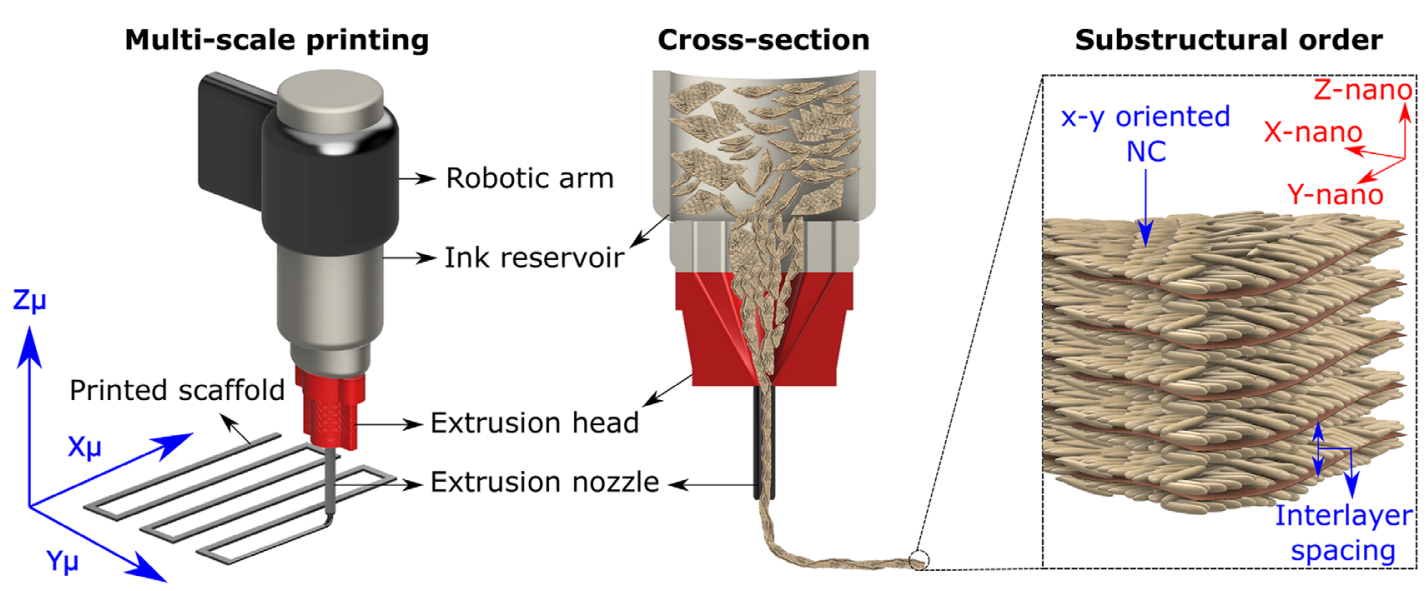

TOC: Multi-scale 3D printing enables the convergence of the microstructure with selforientation of the building block at the nanoscale. 